\title{
Salidroside inhibits NLRP3 inflammasome activation and apoptosis in microglia induced by cerebral ischemia/reperfusion injury by inhibiting the TLR4/NF-кB signaling pathway
}

\author{
Jie Liu ${ }^{1}$, Wei Ma ${ }^{1}$, Cheng-Hao Zang ${ }^{2}$, Guo-Dong Wang ${ }^{1}$, Si-Jia Zhang ${ }^{1}$, Hong-Jie Wu ${ }^{1}$, Ke-Wei Zhu ${ }^{1}$, \\ Xiang-Lin Xiang ${ }^{1}$, Chun-Yan $\mathrm{Li}^{1}$, Kuang-Pin Liu ${ }^{1}$, Jian-Hui Guo ${ }^{2}$, Li-Yan Li ${ }^{1}$ \\ ${ }^{1}$ Institute of Neuroscience, Kunming Medical University, Kunming, China; ${ }^{2}$ Second Department of General Surgery, First People's Hospital of \\ Yunnan Province, Kunming, China \\ Contributions: (I) Conception and design: LY Li, JH Guo; (II) Administrative support: None; (III) Provision of study materials or patients: None; (IV) \\ Collection and assembly of data: J Liu, W Ma, CH Zang, GD Wang, SJ Zhang, HJ Wu; (V) Data analysis and interpretation: KW Zhu, XL Xiang, \\ CY Li, KP Liu; (VI) Manuscript writing: All authors; (VII) Final approval of manuscript: All authors. \\ Correspondence to: Jian-Hui Guo. Second Department of General Surgery, First People's Hospital of Yunnan Province, 157 Jinbi Road, Kunming 650032, \\ China. Email: guojianhuikm@163.com; Li-Yan Li. Institute of Neuroscience, Kunming Medical University, Kunming, China. Email: kmliyanl@163.com.
}

Background: The NOD-like receptor family pyrin domain-containing 3 (NLRP3) inflammasome is an important mediator of neuroinflammatory responses that regulates inflammatory injury following cerebral ischemia and may be a potential target. Salidroside (Sal) has good anti-inflammatory effects; however, it remains unclear whether Sal can regulate NLRP3 inflammasome activation through the Toll-like receptor 4 (TLR4)/ nuclear factor kappa B (NF-кB) signaling pathway after cerebral ischemia to alleviate inflammatory injury.

Methods: We established an oxygen-glucose deprivation and reoxygenation (OGD/R) model of BV2 cells and a middle cerebral artery occlusion/reperfusion (MCAO/R) rat model. Cell Counting Kit-8 (CCK-8), flow cytometry and terminal deoxynucleotidyl transferase dUTP nick-end labeling (TUNEL) assay were used to detect the viability and apoptosis of BV2 cells. Enzyme-linked immunosorbent assay (ELISA) was used to detect the level of inflammatory factors. 2,3,5-triphenyltetrazolium chloride (TTC) staining and modified Neurological Severity Score (mNSS) were used to detect cerebral infarction volume and neurological deficit in rats. Western blot, immunohistochemistry and immunofluorescence staining were used to detect the protein expression levels.

Results: Our results showed that Sal increased viability, inhibited lactate dehydrogenase (LDH) release, and reduced apoptosis in OGD/R-induced BV2 cells. Sal reduced the levels of tumor necrosis factor- $\alpha(\mathrm{TNF}-\alpha)$, interleukin (IL)-6, and IL-8. Following induction by OGD/R, BV2 cells exhibited NLRP3 inflammasome activation and increased protein levels of NLRP3, apoptosis-associated speck-like protein containing a CARD (ASC), caspase-1, IL-1 $\beta$, and IL-18. Protein levels of key TLR4 signaling pathway elements, such as TLR4, myeloid differentiation primary response 88 (MyD88), and phosphorylated nuclear factor kappa B

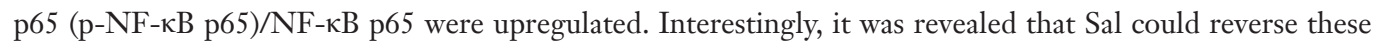
changes. In addition, TAK242, a specific inhibitor of TLR4, had the same effect as Sal treatment on BV2 cells following induction by OGD/R. In the MCAO/R rat model, Sal was also observed to inhibit NLRP3 inflammasome activation in microglia, reduce cerebral infarction volume, and inhibit apoptosis.

Conclusions: In summary, we found that Sal inhibited NLRP3 inflammasome activation and apoptosis in microglia induced by cerebral ischemia/reperfusion injury by inhibiting the TLR4/NF- $\mathrm{kB}$ signaling pathway, thus playing a protective role. Therefore, Sal may be a promising drug for the clinical treatment of ischemic stroke.

Keywords: Salidroside (Sal); microglia; cerebral ischemia/reperfusion injury; NOD-like receptor family pyrin domain-containing 3 (NLRP3) inflammasome; apoptosis

Submitted Oct 09, 2021. Accepted for publication Nov 22, 2021.

doi: $10.21037 /$ atm-21-5752

View this article at: https://dx.doi.org/10.21037/atm-21-5752 


\section{Introduction}

Stroke is a leading cause of disability and death worldwide, the majority of which involve ischemic stroke (1). Although ischemic stroke is a serious threat to human health, the current treatment methods are limited. At present, the leading effective treatment is intravenous thrombolytic therapy, which can restore blood flow but may also lead to oxidative stress, inflammatory response, autophagy, and apoptosis, thereby causing cerebral ischemia/reperfusion (I/R) injury $(2,3)$. Inflammatory responses play a key role in the pathological progression of ischemic stroke and have an important influence on its prognosis (4). After cerebral ischemia, energy depletion and hypoxia lead to nerve cell death. Apoptotic or necrotic neurons activate reactive astrocytes and attract microglia to migrate to the site of inflammation, and these inflammatory cells produce a large number of inflammatory proteins, including pathological and inducible form of nitric oxide synthase, which produces nitrosation stress (5). Inflammatory factors increase infiltration of inflammatory cells, leading to further release of inflammatory mediators and reactive oxygen species, which triggers an inflammatory cascade that leads to neurovascular injury, blood brain barrier injury, and neuronal injury (6). Therefore, inhibiting inflammatory responses may be an important strategy for the treatment of cerebral I/R injury. Microglia, permanent immune cells of the central nervous system, play an important role in inflammatory responses and are the first line of immune defense (7). Microglia are first activated after cerebral ischemia. Activated microglia secrete a variety of inflammatory cytokines, including tumor necrosis factor- $\alpha$ (TNF- $\alpha$ ) and interleukin (IL)-6, as well as cell adhesion molecules and proteases, which can promote a strong inflammatory response and lead to tissue damage $(8,9)$. In addition, microglia also help to synchronize neuronal activity and plasticity, thereby enhancing the recovery of neurological function after stroke (9). Previous studies have shown that the status microglia determines the inflammatory response of brain tissue (10), and microglial dysfunction and death are associated with an uncontrolled inflammatory response. Overactivation and apoptosis of microglia are reportedly related to inflammation and injury in ischemic cerebrovascular disease (11). Microglia are activated and damaged under ischemic and hypoxic conditions, causing an inflammatory response (12). However, inhibition of the inflammatory response of microglia can reduce their apoptosis (13). In summary, microglia-mediated inflammatory responses play a key role in the occurrence, development, and outcome of ischemic stroke (14); accordingly, it may be possible to treat ischemic stroke by inhibiting microglial overactivation and apoptosis.

The NOD-like receptor family pyrin domain-containing 3 (NLRP3) inflammasome is an important mediator of neuroinflammatory response and mediates inflammatory injury following cerebral ischemia (15). Martinon first discovered and proposed the concept of inflammasomes in 2002 (16). The NLRP3 inflammasome consists of the NLRP3 receptor, apoptosis-associated speck-like protein containing a CARD (ASC), and caspase-1, and is one of the most widely studied inflammasomes (17). It is an important regulator of inflammation because it activates caspase 1 and production of pro-inflammatory cytokines IL-18 and IL-1 $\beta$, leading to an inflammatory response (18). In addition, the NLRP3 inflammasome plays an important role in the pathological progression of ischemic stroke (19). Inhibiting activity of the NLRP3 inflammasome can reduce the inflammatory injury elicited by cerebral I/R (20). Activation of the NLRP3 inflammasome involves the interaction of multiple signaling pathways and complexes, and serves as a key molecular pathway in the inflammatory cascade (21). A previous study suggested that activation of the Tolllike receptor 4 (TLR4)/nuclear factor kappa B (NF-kB) signaling pathway is a basic step in the formation of NLRP3 inflammasomes and closely related to their activation (22). A transmembrane receptor protein of the innate immune system, TLR4 is upregulated after cerebral I/R injury. Upregulation of TLR4 activates NF- $\mathrm{NB}$, which induces the release of many pro-inflammatory factors and triggers an inflammatory response that can lead to brain injury (23). Inhibition of the TLR4/NF- $\mathrm{B}$ signaling pathway can inhibit inflammatory responses and apoptosis after cerebral I/R injury (24). Therefore, we hypothesized that inhibiting activation of the NLRP3 inflammasome might be an ideal target for the treatment of cerebral ischemic inflammatory injury.

Salidroside (Sal), a phenylpropanoside extracted from Rhodiola rosea, has anti-inflammatory, anti-oxidative stress, anti-apoptosis, and other pharmacological effects (25). Sal was previously shown to exert a neuroprotective antiinflammatory effect in ischemic stroke, but the underlying mechanism is still unclear. It can reduce inflammation and brain injury in ischemic stroke by regulating the phosphoinositide 3 kinase/protein kinase $\mathrm{B} /$ nuclear factor erythroid 2-related factor $2 / \mathrm{NF}-\kappa \mathrm{B}$ signaling pathway (26). One study reported that Sal alleviates diabetic neuropathic 
pain through regulation of the AMP-activated protein kinase-NLRP3 inflammasome axis (27). A previous study showed that microglia are the main source of NLRP3 in the central nervous system (28). Accordingly, microglia are often used to study inflammatory responses and evaluate involvement of the NLRP3 inflammasome in pathological processes of ischemic stroke (29). Therefore, based on the important role of microglia in cerebral I/R inflammatory injury. In cerebral I/R injury, whether Sal can affect inflammatory injury by regulating the activation of NLRP3 inflammasome in microglia, and whether TLR4/ $\mathrm{NF}-\kappa \mathrm{B}$ signaling pathway is involved in this regulatory process is still unclear, and further research is needed. In addition, since the status of microglia is related to the inflammatory response of brain tissue, whether Sal affects the apoptosis of microglia in cerebral I/R injury and its regulatory mechanism also remains to be further explored. Therefore, in this study, an oxygen-glucose deprivation and reoxygenation (OGD/R) model in BV2 cells and a middle cerebral artery occlusion/reperfusion (MCAO/R) rat model were established. Our results confirmed that Sal could inhibit NLRP3 inflammasome activation and apoptosis in microglia induced by cerebral I/R injury by inhibiting the TLR4/NF- $\mathrm{B}$ signaling pathway, thus providing a new therapeutic target for cerebral I/R injury.

We present the following article in accordance with the ARRIVE reporting checklist (available at https://dx.doi. org/10.21037/atm-21-5752).

\section{Methods}

\section{Chemicals and reagents}

Salidroside (purity $\geq 98 \%$ ) was purchased from Chengdu Munster Biotechnology Co., Ltd. (Chengdu, China). A mouse microglia cell line (BV2) was purchased from Kunming Cell Bank of the Type Culture Collection Committee of the Chinese Academy of Sciences (Kunming, China). The following primary antibodies were used: rabbit anti-NLRP3 [15101, Cell Signaling Technology (CST), Danvers, MA, USA], rabbit anti-ASC (67824, CST), rabbit anti-caspase-1 (AB138483, Abcam, Cambridge, UK), rabbit anti-IL-1ß (16806-1-AP, Proteintech, Rosemont, IL, USA), rabbit anti-IL-18 (AB207323, Abcam), rabbit-Bcl-2 (AB194583, Abcam), rabbit anti-Bax (2772S, CST), rabbit anti-cleaved caspase-3 (9664, CST), mouse anti-TLR4 (SC-293072, Santa Cruz Biotechnology, Dallas, TX, USA), rabbit anti-MyD88 (4283S, CST), rabbit anti-p-NF-кB p65
(PhosphoS536; AB86299, Abcam), rabbit anti-NF-кB p65 (AB16502, Abcam), rabbit anti-NLRP3 (NBP2-12446SS, Novus, USA), Goat anti-IBA-1(NB100-1028, Novus), and rabbit anti- $\beta$-actin (GB11001, ServiceBio, Wuhan, China). The following secondary antibodies were used: goat antirabbit IgG (L3012, Signalway Antibody, College Park, MD, USA), goat anti-mouse IgG (L3032, Signalway), Cy3 conjugated Donkey Anti-Goat IgG (GB21404, ServiceBio), fluorescein isothiocyanate (FITC) conjugated Donkey Anti-Rabbit IgG (GB22403, ServiceBio). We obtained TAK242 from Selleck Chemicals (S7455, Resatorvid; Shanghai, China) and Dimethyl sulfoxide (DMSO; D8371, Solarbio, Beijing, China). Enzyme-linked immunosorbent assay (ELISA) was performed with kits for mouse tumor necrosis factor- $\alpha$ (TNF- $\alpha$; 1217202, Dakewe, Shenzhen, China), mouse IL-6 (1210602, Dakewe), and mouse IL-8 (CME0008, 4A Biotech, Beijing, China).

\section{Cell culture and OGD/R model}

The BV2 cells were cultured in Dulbecco's Modified Eagle's Medium (DMEM) with high glucose (SH30243.01, Hyclone, Logan, UT, USA) containing 10\% fetal bovine serum (FBS; 04-001-1ACS, Biological Industries, BeitHaemek, Israel), penicillin (100 IU/mL), and streptomycin $(100 \mu \mathrm{g} / \mathrm{mL})$. Cells were cultured in a humidified incubator at $37^{\circ} \mathrm{C}$ and $5 \% \mathrm{CO}_{2}$, and the medium was changed every 2-3 days. During the logarithmic growth phase of BV2 cells, the cell medium was replaced with DMEM without glucose (90113, Solarbio, Beijing, China). Cells were placed in anoxic chambers containing $5 \% \mathrm{CO}_{2}$ and $95 \% \mathrm{~N}_{2}$, which were placed in an incubator for $1,2,3,4,6$, or $8 \mathrm{~h}$ to establish an OGD model. At the end of OGD, the medium was replaced with high-glucose DMEM for subsequent culture in an aerobic incubator for $12 \mathrm{~h}$ to establish the OGD/R model in BV2 cells. Cells in the control group were always cultured under normal conditions. Sal was dissolved in water at various concentrations before co-incubation with OGD/R-induced BV2 cells. On the basis of literature reports (30), in order to explore the effective concentration of Sal that could improve the viability of BV2 cells, OGD/ $\mathrm{R}$-induced BV2 cells were co-incubated with 0, 6.25, 12.5, 25,50 , or $100 \mu \mathrm{M} \mathrm{Sal}$, and viability was detected by CCK8 assay. Therefore, the drug concentration that improves the vitality of BV2 cells is selected. In addition, TAK242, a specific TLR4 inhibitor, was dissolved in DMSO and medium at a concentration of $1 \mu \mathrm{M}$ (31). Cells in OGD/R + TAK242 group were co-incubated with TAK242, and cells 


\section{Page 4 of 16}

in control group and OGD/R group were co-incubated with the same volume of corresponding solvent.

\section{Cell viability assay}

The BV2 cells $\left(1 \times 10^{4} /\right.$ well $)$ were seeded into 96 -well plates for assessment of viability with a Cell Counting Kit- 8 (CCK-8; C0038, Beyotime, Shanghai, China) according to the manufacturer's instructions. The absorbance of each well was measured at $450 \mathrm{~nm}$ with a microplate reader.

\section{Lactate dehydrogenase (LDH) release assay}

An LDH cytotoxicity detection kit (A020-2, Nanjing Jiancheng Bioengineering Institute, Nanjing, China) was used to evaluate cell damage. The LDH activity in the culture medium supernatant was measured at $450 \mathrm{~nm}$ with a microplate reader.

\section{Annexin V-FITC apoptosis detection}

Cell apoptosis was determined using an Annexin V-FITC Apoptosis Detection Kit (KGA105-KGA10, Keygen Biotechnology, Nanjing, China) according to the manufacturer's guidelines. A FACSCelesta ${ }^{\mathrm{TM}}$ flow cytometer (Becton, Dickinson, and Co., Franklin Lakes, NJ, USA) was used for detection.

\section{ELISA}

The supernatant of BV2 cell culture medium was collected. Concentrations of TNF- $\alpha$, IL- 6 , and IL- 8 in culture supernatants were determined with specific ELISA kits. Absorbances were measured at $450 \mathrm{~nm}$ with a microplate reader.

\section{Animals and treatment}

Adult male Sprague-Dawley (SD) rats, weighing 200-250 g (7-8 weeks old), were provided by the Experimental Animal Center of Kunming Medical University. Animals were housed in a standardized animal care center with proper temperature and humidity and a $12 \mathrm{~h}$ light/dark cycle. The rats were given free access to food and water. All rat experiments were approved by the Animal Research Ethics Committee of Kunming Medical University (Kmmu202100001410) and carried out in accordance with the Guide for the Care and Use of Laboratory Animals, 8th edition (32). A total of 42 rats were randomly divided into 3 groups: sham group, $\mathrm{MCAO} / \mathrm{R}$ group, and $\mathrm{MCAO} / \mathrm{R}+$ Sal group. According to previous studies (33), rats in the $\mathrm{MCAO} / \mathrm{R}+$ Sal group were intraperitoneally injected with Sal $(50 \mathrm{mg} / \mathrm{kg}$, dissolved in normal saline) immediately after surgery of inserting the suture, and rats in the sham group and MCAO/R group were intraperitoneally injected with the same amount of normal saline, for consecutive 7 days.

\section{MCAO/R model}

The MCAO model was constructed based on the improved Longa method. The rats were anesthetized by intraperitoneal injection of $1 \%$ pentobarbital sodium solution $(30 \mathrm{mg} / \mathrm{kg})$, and their necks were disinfected. A median incision was made to expose the carotid artery and separate the right common carotid artery (CCA), internal carotid artery (ICA), and external carotid artery (ECA). The ECA and CCA were ligated with silk thread and ICA was temporarily occluded. An incision was made in the CCA and nylon monofilament sutures were inserted into the ICA, from which they were slowly advanced to the middle cerebral artery (MCA). The same procedure was performed in the sham group, except that the sutures were not plugged into the ICA. At $2 \mathrm{~h}$ after occlusion, the nylon sutures were removed for reperfusion. During surgery and ischemia, the body temperature of rats was maintained at $37.0 \pm 0.5^{\circ} \mathrm{C}$ by heating pad. A total of 5 rats died before sampling and testing due to possible reasons such as anesthesia or surgical injury and were not included in the analysis.

\section{Evaluation of infarct volume}

After 7 days of cerebral ischemia and reperfusion, the rat brains were removed, frozen at $-20{ }^{\circ} \mathrm{C}$ for $20 \mathrm{~min}$, and cut into 6 pieces. Brain slices were then immersed in preheated 2\% 2,3,5-triphenyltetrazolium chloride (TTC; T8877, Sigma Aldrich, St. Louis, MO, USA) solution, and incubated at $37{ }^{\circ} \mathrm{C}$ for $20 \mathrm{~min}$, then fixed with $4 \%$ paraformaldehyde for $30 \mathrm{~min}$. Infarct volume was detected by ImageJ software (https://imagej.nih.gov/ij/), and the data were expressed as the percentage of infarct volume in the total volume of brain tissue.

\section{Evaluation of neurological deficits}

Neurological deficits were assessed using the modified Neurological Severity Score (mNSS) on day 7 after surgery. 
The mNSS involves the assessment of multiple tasks such as movement and balance, with a maximum defect score of 18 . All rats were pretrained for 7 days before surgery.

\section{Terminal deoxynucleotidyl transferase dUTP nick-end labeling assay}

According to the manufacturer's guidelines, apoptotic cells were detected by terminal deoxynucleotidyl transferase dUTP nick-end labeling (TUNEL) assay using an apoptosis detection kit (G1501, ServiceBio). Images were collected using an inverted fluorescence microscope (ZEISS Axio Observer Z1, Oberkochen, Germany). Numbers of TUNEL-positive cells and total cells were counted in 5 random areas. Ratios of apoptosis were calculated as follows: number of TUNEL-positive cells/total number of cells in each area $\times 100 \%$.

\section{Immunobistochemical (IHC) staining}

Briefly, BV2 cells were collected and fixed with 4\% paraformaldehyde, embedded in dehydrated paraffin, and cut into $4 \mu \mathrm{m}$ thick sections for IHC staining. The brain tissue of rats was prepared into paraffin sections. Subsequently, sections were dewaxed with xylene and subjected to antigenic repair with ethylenediamine tetraacetic acid (EDTA; $\mathrm{pH}=8.0$ ) antigenic repair solution. After blocking endogenous peroxidases with $3 \%$ hydrogen peroxide for $30 \mathrm{~min}$, sections were incubated with primary antibodies against NLRP3 (1:300), caspase-1 (1:500), and TLR4 $(1: 250)$ overnight at $4{ }^{\circ} \mathrm{C}$. The following day, sections were incubated with an appropriate secondary antibody (1:350) for $50 \mathrm{~min}$ at room temperature. Next, sections were stained with a 3,3'-diaminobenzidine (DAB) colorimetric kit and hematoxylin. After dehydration and drying, neutral gum was applied to seal sections. Images were collected using a CKX53 inverted fluorescence microscope (Olympus, Tokyo, Japan), and percentages of positive staining (brown) were measured with ImageJ.

\section{Immunofluorescence staining}

The brains of rats were removed after cardiac perfusion with $4 \%$ paraformaldehyde, and sliced into $4 \mu \mathrm{m}$ slices. Sections were sealed with $5 \%$ goat serum and incubated overnight with NLRP3 antibody (1:50) and IBA-1 antibody $(1: 50)$ at $4{ }^{\circ} \mathrm{C}$, and then with donkey anti-rabbit IgG (1:100), donkey anti-goat $\operatorname{IgG}(1: 200)$ were incubated at $37^{\circ} \mathrm{C}$ for
$1 \mathrm{~h}$ and 4',6-diamidino-2-phenylindole (DAPI) for $5 \mathrm{~min}$. Images were collected using an inverted fluorescence microscope (ZEISS Axio Observer Z1) and the fluorescence intensity was analyzed with ImageJ software.

\section{Western blot}

Protein extracts from each group of cells were isolated with radioimmunoprecipitation assay (RIPA) protein lysis buffer. Protein concentrations were determined by bicinchoninic acid (BCA) assay (P0010, Beyotime), and equivalent protein samples $(25 \mu \mathrm{g})$ were separated by $12 \%$ BIS-Tris sodium dodecyl sulfate polyacrylamide gel electrophoresis (SDS-PAGE) and transferred to polyvinylidene fluoride (PVDF) membranes. After blocking with 5\% skim milk at room temperature for $1 \mathrm{~h}$, membranes were incubated overnight at $4{ }^{\circ} \mathrm{C}$ with primary antibodies against $\mathrm{Bcl}-$ 2 (1:1,000), Bax $(1: 1,000)$, cleaved caspase-3 $(1: 1,000)$, TLR4 (1:1,000), MyD88 (1:1,000), p-NF-кB p65 (1:1,000), NF-кB p65 (1:1,000), NLRP3 (1:1,000), ASC $(1: 1,000)$, caspase-1 (1:1,000), IL-1 $\beta$ (1:1,000), IL-18 $(1: 1,000)$, and $\beta$-actin $(1: 1,000)$. Finally, membranes were incubated with appropriate secondary antibodies $(1: 5,000)$ at room temperature for $2 \mathrm{~h}$. Protein electrophoresis bands were captured following the addition of an enhanced chemiluminescence (ECL) developer and analyzed by ImageJ software.

\section{Statistical analysis}

Statistical analysis was performed using Prism software (version 6.0, GraphPad Software, San Diego, CA, USA). Data were obtained from 3 independent experiments and expressed as mean \pm standard deviation (SD). Oneway analysis of variance (ANOVA) was used, followed by Bonferroni post-hoc test between groups. Statistical significance was considered when $\mathrm{P}<0.05$.

\section{Results}

\section{Sal increased the viability of $B V 2$ cells following induction by $O G D / R$ and reduced $L D H$ release}

To establish a suitable OGD/R model of BV2 cells, BV2 cells were first exposed to OGD for $1,2,3,4,6$, or $8 \mathrm{~h}$, and then reoxygenated for $12 \mathrm{~h}$. The CCK- 8 assay results (Figure $1 A$ ) revealed that OGD $4 \mathrm{~h} / \mathrm{R} 12 \mathrm{~h}$ significantly decreased cell viability, while OGD 6 h/R 12 h and OGD 

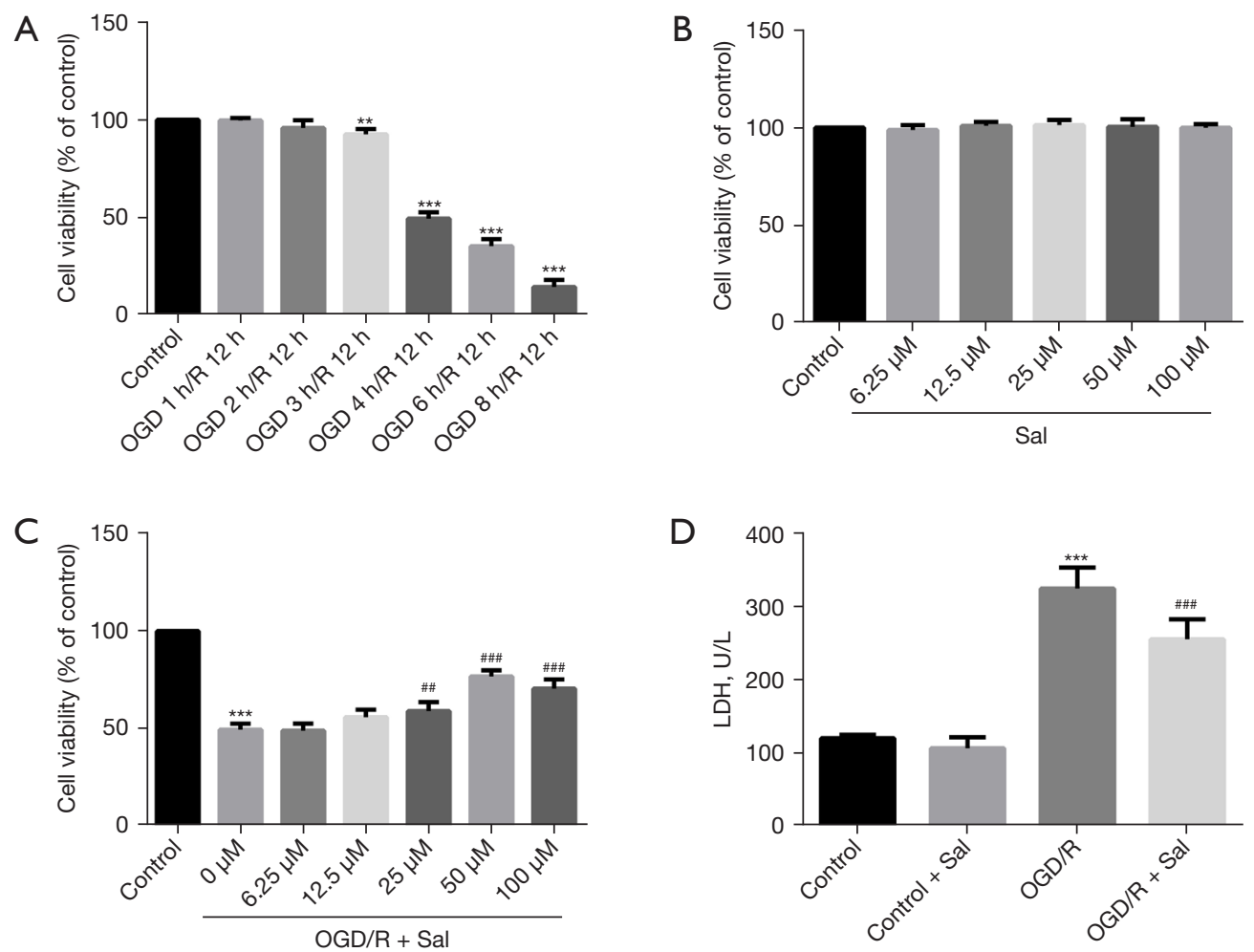

Figure 1 Sal increased the viability of BV2 cells following induction by OGD/R and reduced LDH release. (A) BV2 cells were OGD for 1, 2, 3, 4, 6, or $8 \mathrm{~h}$, and then reoxygenated (R) for $12 \mathrm{~h}$. A CCK-8 assay was used to detect cell viability (n=6). (B) Normal BV2 cells were cultured with various concentrations of Sal $(0,6.25,12.5,25,50$, and $100 \mu \mathrm{M})$, and cell viability was detected by CCK-8 assay (n=6). (C) BV2 cells were OGD for $4 \mathrm{~h}$ and then reoxygenated for $12 \mathrm{~h}$ (OGD $4 \mathrm{~h} / \mathrm{R} 12 \mathrm{~h}$ ) to establish a cellular OGD/R model. BV2 cells were incubated with various concentrations $(0,6.25,12.5,25,50$, or $100 \mu \mathrm{M})$ of Sal during the process of establishing the OGD/R model. A CCK-8 assay was used to detect cell viability (n=6). (D) BV2 cells induced by OGD $4 \mathrm{~h} / \mathrm{R} 12 \mathrm{~h}$ were incubated with $50 \mu \mathrm{M}$ Sal, and then the amounts of $\mathrm{LDH}$ released were evaluated $(\mathrm{n}=6)$. Data are expressed as mean $\pm \mathrm{SD}$. ${ }^{* *} \mathrm{P}<0.01,{ }^{* * *} \mathrm{P}<0.001$ compared with control group; ${ }^{\# \#} \mathrm{P}<0.01$, ${ }^{\# \# \#} \mathrm{P}<0.001$ compared with OGD/R group. OGD, oxygen-glucose deprivation; Sal, salidroside; CCK-8, Cell Counting Kit-8; LDH, lactate dehydrogenase; SD, standard deviation.

8 h/R 12 h more significantly decreased viability, suggesting excessive cell injury. Therefore, OGD $4 \mathrm{~h} / \mathrm{R} 12 \mathrm{~h}$ was selected to establish the OGD/R cell model for subsequent experiments. To evaluate the toxicity of Sal to BV2 cells, normal cultured BV2 cells were co-incubated with $0,6.25$, $12.5,25,50$, or $100 \mu \mathrm{M} \mathrm{Sal}$, and viability was detected by CCK-8 assay (Figure 1B). The results showed no significant changes in cell viability, suggested that Sal had no obvious cytotoxic effect on normal cultured BV2 cells. To explore the effective concentration of Sal that could improve the viability of BV2 cells, OGD/R-induced BV2 cells were coincubated with $0,6.25,12.5,25,50$, or $100 \mu M$ Sal, and viability was detected by CCK-8 assay (Figure 1C). The results showed that BV2 cell viability was significantly decreased after OGD/R induction, whereas co-incubation with 25,50 , or $100 \mu \mathrm{M}$ Sal could improve cell viability. Among the tested concentrations, cell viability was most obviously improved with $50 \mu \mathrm{M}$ Sal. Therefore, we chose $50 \mu \mathrm{M}$ as an effective drug concentration for follow-up experiments. Next, we incubated OGD/R-induced BV2 cells with $50 \mu \mathrm{M}$ Sal and detected levels of LDH release (Figure 1D). The results showed that LDH release by BV2 cells treated with Sal was significantly lower than that of the OGD/R group, further indicating that Sal could reduce cell damage. Collectively, these results suggest that Sal had a protective effect on BV2 cells following induction by OGD/R.

Sal inbibited OGD/R-induced apoptosis of BV2 cells 
A
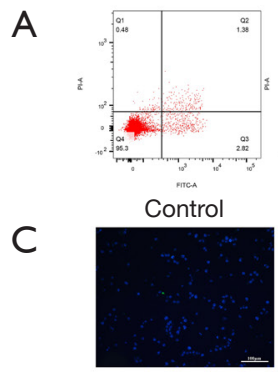

Control

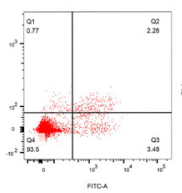

Control + Sal

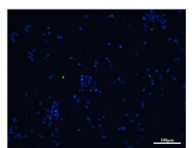

Control + Sal

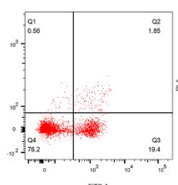

$\mathrm{OGD} / \mathrm{R}$

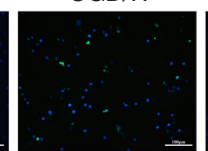

OGD/R

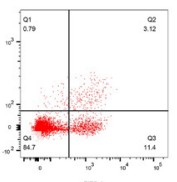

OGD/R + Sal

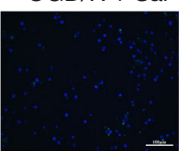

$\mathrm{OGD} / \mathrm{R}+\mathrm{Sal}$
B
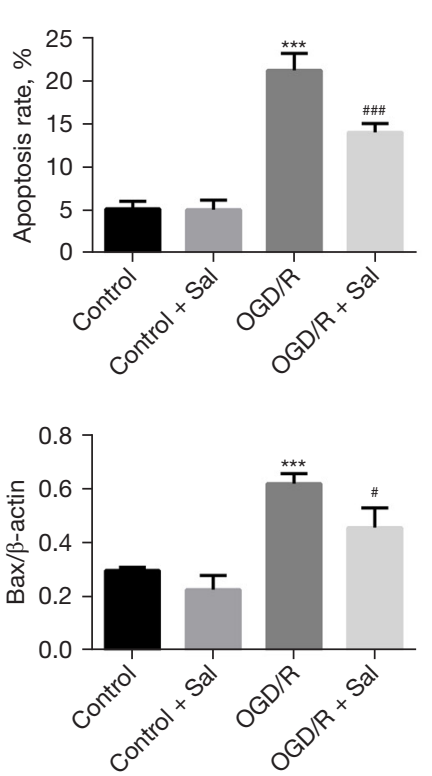
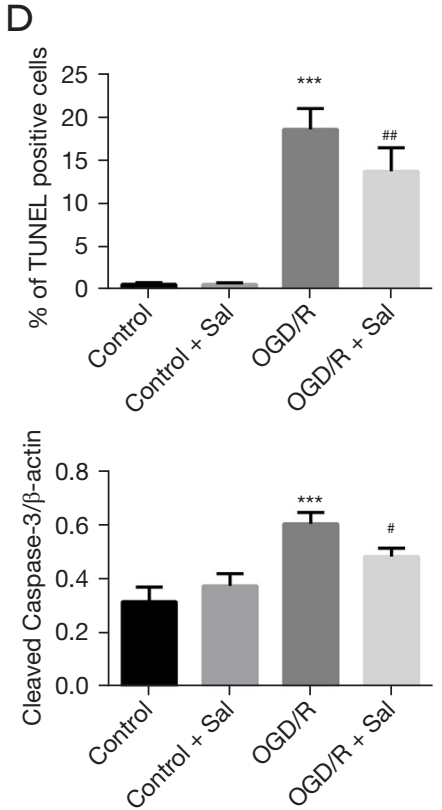

Figure 2 Sal inhibited OGD/R-induced apoptosis of BV2 cells. (A) Apoptosis of BV2 cells was detected by flow cytometry; (B) percentages of apoptotic cells were quantitatively analyzed $(n=4) ;(C)$ representative fluorescent images of TUNEL staining (green) and DAPI (blue)

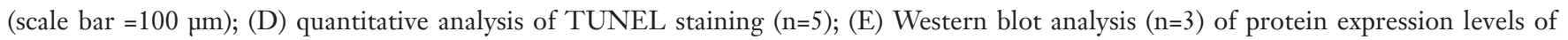
cleaved caspase-3, Bax, and Bcl-2. Data are expressed as mean $\pm \mathrm{SD}$. ${ }^{* * *} \mathrm{P}<0.001$ compared with control group; ${ }^{*} \mathrm{P}<0.05,{ }^{{ }^{* \prime \prime}} \mathrm{P}<0.01,{ }^{{ }^{* \prime \prime \prime \prime}} \mathrm{P}<0.001$ compared with OGD/R group. OGD, oxygen and glucose deprivation; TUNEL, terminal deoxynucleotidyl transferase dUTP nick-end labeling assay; SD, standard deviation.

To further explore whether Sal had a protective effect on $\mathrm{BV} 2$ cells induced by OGD/R, apoptosis was detected by flow cytometry (Figure $2 A, 2 B$ ) and TUNEL staining (Figure 2C,2D). The results showed that compared with the model group, treatment with Sal significantly reduced the percentage of apoptotic BV2 cells following induction by OGD/R. In addition, western blot (Figure 2E) to detect expression levels of apoptotic proteins showed that, compared with the control group, expression levels of apoptotic proteins Bax and cleaved caspase- 3 were increased in the OGD/R group, whereas expression of the anti-apoptotic protein Bcl-2 was decreased. However, this change could be reversed by Sal treatment. In the OGD/ $\mathrm{R}+$ Sal group, expression of Bax and cleaved caspase- 3 was significantly decreased, while that of Bcl-2 expression was significantly increased. There was no statistically significant difference between the control + Sal group and the control group. These results further demonstrate that Sal inhibited the apoptosis of BV2 cells.

\section{Sal reduced the release of inflammatory factors from}

\section{OGD/R-induced BV2 cells}

To examine whether Sal could inhibit the inflammatory response of OGD/R-induced BV2 cells, ELISA (Figure 3) was performed to detect levels of several major inflammatory factors in cell culture medium supernatants. The results demonstrated that release of TNF- $\alpha$, IL- 6 , and IL- 8 was significantly increased in the OGD/R group compared with the control group, but significantly decreased in the OGD/ $\mathrm{R}+$ Sal group compared with the OGD/R group after treatment. There was no statistically significant difference between the control + Sal group and the control group. These findings suggest that Sal inhibited the inflammatory response of BV2 cells following induction by OGD/R.

\section{Sal inbibited NLRP3 inflammasome activation in OGD/ R-induced BV2 cells}

To evaluate whether Sal had a regulatory effect on NLRP3 inflammasome activation in BV2 cells following induction by OGD/R, expression levels of several key molecules to 

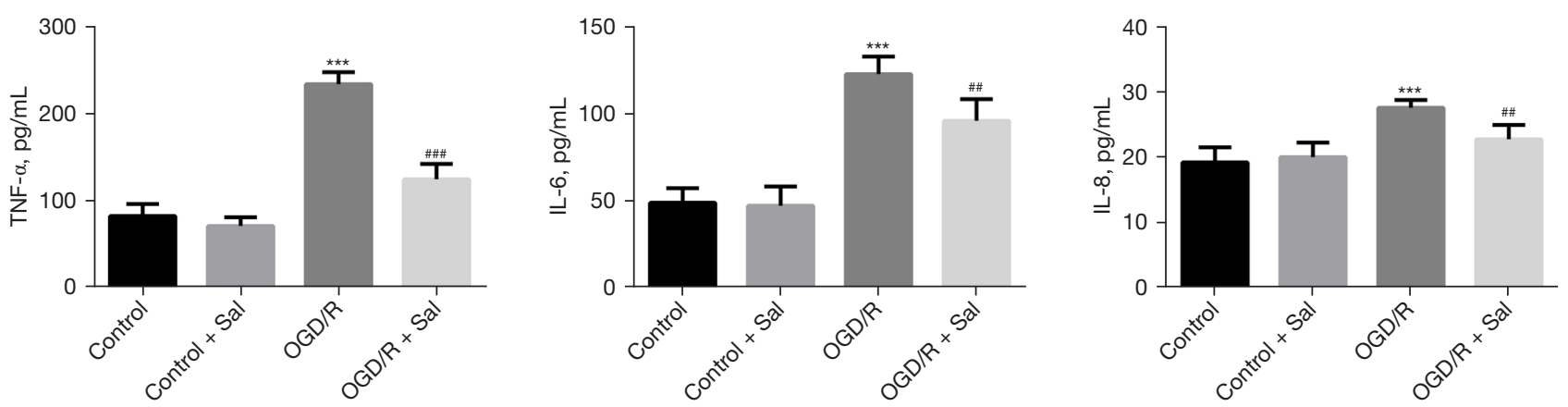

Figure 3 Sal reduced the release of inflammatory factors from OGD/R-induced BV2 cells. TNF- $\alpha$, IL-6, and IL-8 in culture medium supernatants were determined by ELISA ( $\mathrm{n}=6)$. Data are expressed as mean $\pm \mathrm{SD} .{ }^{* * *} \mathrm{P}<0.001$ compared with control group; ${ }^{\# \#} \mathrm{P}<0.01$,

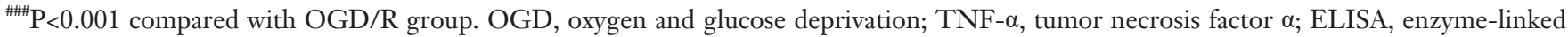
immunosorbent assay; SD, standard deviation.

NLRP3 activation were detected by western blot (Figure $4 A$ ). Compared with the control group, expression levels of NLRP3, ASC, caspase-1, IL-1 $\beta$, and IL-18 were increased after OGD/R induction, indicating activation of NLRP3 inflammasomes. However, $50 \mu \mathrm{M}$ Sal significantly reduced the expression levels of these molecules. There was no statistically significant difference between the control + Sal group and the control group. The IHC staining, which was used to further detect the expression levels of NLRP3 (Figure 4B,4C) and caspase-1 (Figure 4D,4E), showed that levels of both proteins were significantly lower in the OGD/ $\mathrm{R}+$ Sal group compared with the OGD/R group, consistent with western blotting results. These findings suggest that Sal could inhibit NLRP3 inflammasome activation in OGD/ $\mathrm{R}$-induced BV2 cells to inhibit the inflammatory response.

\section{Sal inbibited TLR4/NF- $\mathrm{KB}$ signaling patbway activation in OGD/R-induced BV2 cells}

To evaluate whether Sal could regulate the TLR4/NF-кB signaling pathway, expression levels of several key pathwayrelated molecules were detected by western blot (Figure 5A). Compared with the control group, expression levels of

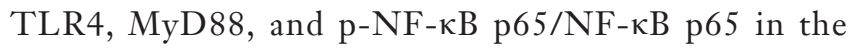
OGD/R group were significantly increased. These results indicate that the TLR4 signaling pathway was activated in BV2 cells following induction by OGD/R. However, these changes were reversed by Sal treatment. Compared with the OGD/R group, protein expression levels of TLR4, MyD88, and $\mathrm{p}-\mathrm{NF}-\kappa \mathrm{B}$ p $65 / \mathrm{NF}-\kappa \mathrm{B}$ p 65 were decreased in the $\mathrm{OGD} / \mathrm{R}+$ Sal group. There was no statistically significant difference between the control + Sal group and the control group. The results of further detection of TLR4 expression by IHC (Figure 5B, 5C) were consistent with western blot results, suggesting that $\mathrm{Sal}$ could inhibit TLR4/NF- $\mathrm{BB}$ signaling pathway activation in OGD/R-induced BV2 cells.

\section{Inbibition of TLR4 reduced NLRP3 inflammasome activation and apoptosis in OGD/R-induced BV2 cells by inbibiting the TLR4/NF- $\mathrm{KB}$ signaling patbway}

To further verify whether the TLR4/NF- $\mathrm{B}$ signaling pathway could regulate activation of the NLRP3 inflammasome and apoptosis, OGD/R-induced BV2 cells were incubated with the TLR4-specific inhibitor TAK242 to inhibit TLR4 expression. Activation of the TLR4/NF$\mathrm{\kappa B}$ signaling pathway, NLRP3 inflammasome, and apoptosis in BV2 cells were observed, and expression levels of related proteins were detected by western blot. Compared with the OGD/R group, expression levels of TLR4, MyD88, and $\mathrm{p}-\mathrm{NF}-\kappa \mathrm{B}$ p65/NF- $\mathrm{\kappa B}$ p65 were significantly decreased in the OGD/R + TAK242 group, indicating that the TLR4 signaling pathway was inhibited by TAK242 (Figure 6A). Compared with the OGD/R group, protein expression levels of NLRP3, ASC, caspase-1, IL-1 $\beta$, and IL-18 in the OGD/ $\mathrm{R}+$ TAK242 group were significantly decreased (Figure 6B). Expression of apoptotic proteins Bax and cleaved caspase-3 was significantly decreased in the OGD/R + TAK242 group, while that of the anti-apoptotic protein Bcl-2 was significantly increased (Figure 6C). There was a statistically significant difference between the OGD/R + TAK242 group and the OGD/R group. These results indicate that inhibition of TLR4 signaling pathway can inhibit NLRP3 inflammasome activation and apoptosis in BV2 cells 
A

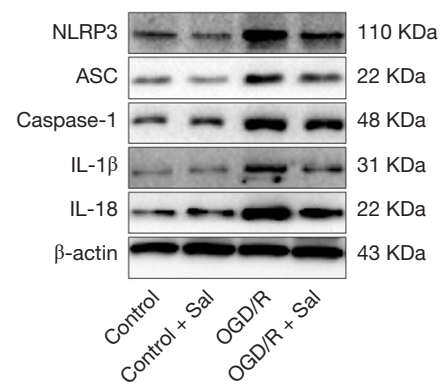

B
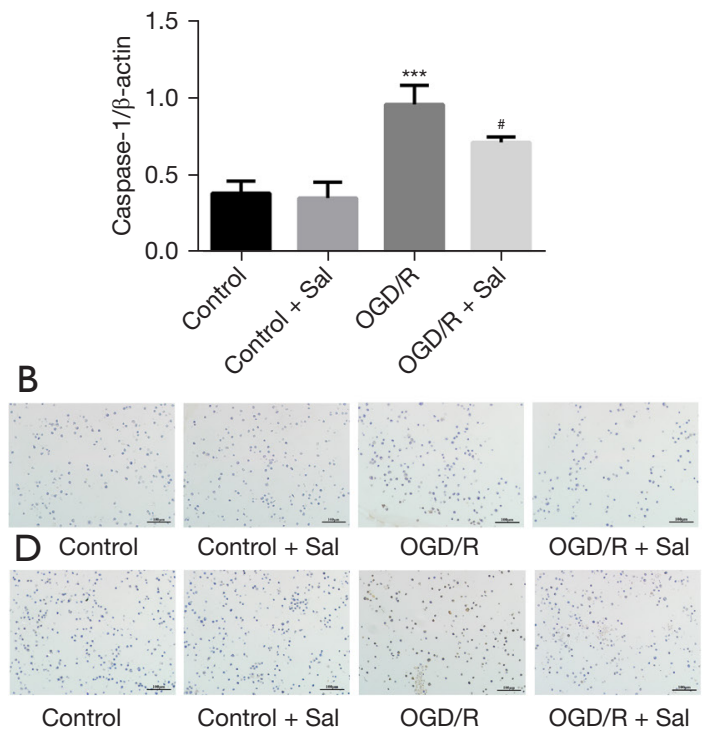
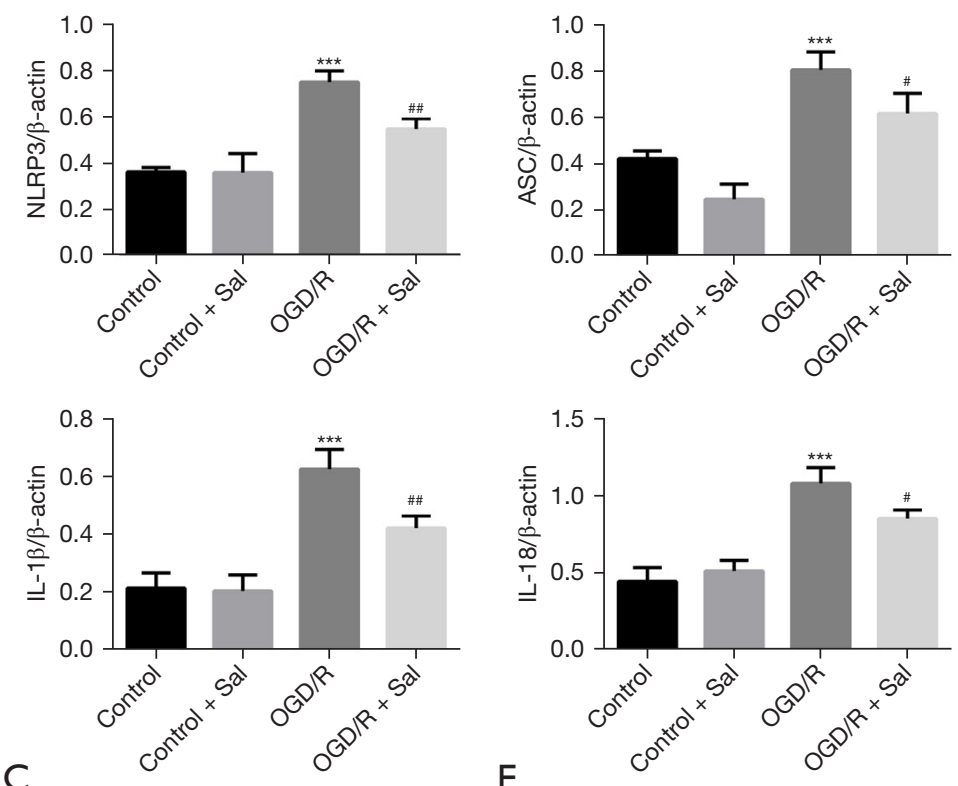

C
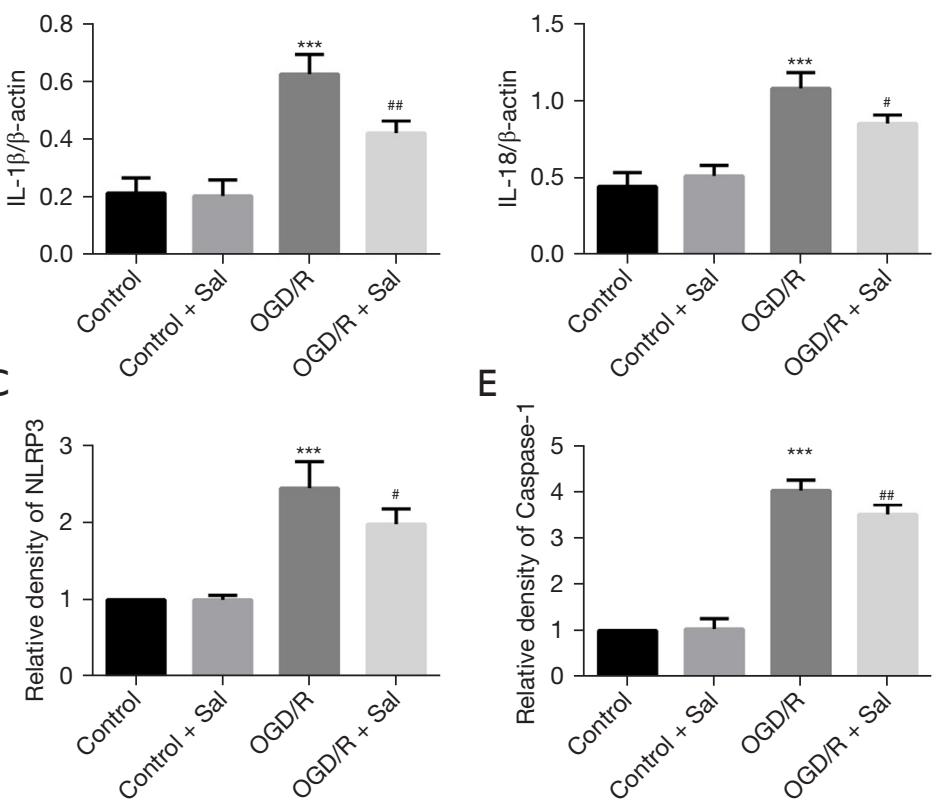

$\mathrm{E}$

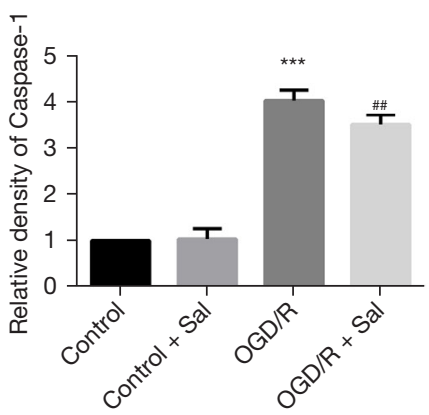

Figure 4 Sal inhibited NLRP3 inflammasome activation in OGD/R-induced BV2 cells. (A) Protein expression levels of NLRP3, ASC, caspase-1, IL-1 $\beta$, and IL-18 were detected by western blot analysis $(n=3)$; representative bands are shown in the figure. Protein expression levels of both NLRP3 (B) and caspase-1 (D) were detected by IHC staining (scale bar $=100 \mu \mathrm{m}$ ). (C,E) Quantitative analysis of IHC staining results $(\mathrm{n}=5)$. Data are expressed as mean $\pm \mathrm{SD}$. ${ }^{* * *} \mathrm{P}<0.001$ compared with control group; ${ }^{\#} \mathrm{P}<0.05,{ }^{\# \#} \mathrm{P}<0.01$ compared with OGD/R group. OGD, oxygen and glucose deprivation; ASC, apoptosis-associated speck-like protein containing a CARD; NLRP3, NOD-like receptor family pyrin domain-containing 3; IHC, immunohistochemical; SD, standard deviation.

following induction by OGD/R. In conclusion, the specific inhibitor of TLR4, TAK242, may inhibit OGD/R-induced NLRP3 inflammasome activation and apoptosis in BV2 cells, consistent with the results of Sal treatment. Therefore, we speculate that Sal regulates NLRP3 inflammasome activation and apoptosis in OGD/R-induced BV2 cells by regulating the TLR4/NF- $\mathrm{KB}$ signaling pathway.

\section{Sal inbibited brain injury induced by MCAO/R in rats and inbibited the NLRP3 inflammasomes activation in microglia}

To further verify the inhibitory effect of Sal on cell apoptosis and activation of NLRP3 inflammasome in microglia, we constructed a rat MCAO/R model and performed related tests after 7 days of treatment with Sal. The TTC staining (Figure $7 A, 7 B$ ) and the mNSS (Figure $7 C$ ) indicated that compared with the $\mathrm{MCAO} / \mathrm{R}$ group, cerebral infarction volume and the mNSS were decreased in the MCAO/R + Sal group.These results suggest that $\mathrm{Sal}$ can reduce cerebral infarction volume and neurological deficits in MCAO/R rats. The TUNEL staining (Figure $7 D, 7 E$ ) indicated that compared with $\mathrm{MCAO} / \mathrm{R}$ group, apoptosis was reduced in the $\mathrm{MCAO} / \mathrm{R}+\mathrm{Sal}$ group. These results indicate that Sal can reduce the apoptosis of brain cells in $\mathrm{MCAO} / \mathrm{R}$ rats, and we speculate that Sal may also reduce the apoptosis of microglia. Considering the above results, it can be concluded that Sal can inhibit brain injury in MCAO/R rats. The IHC 


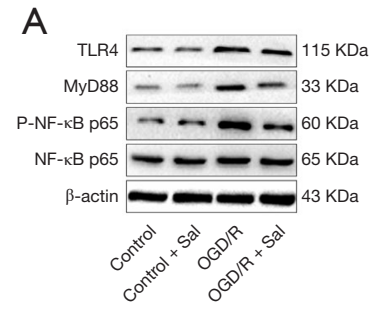

B
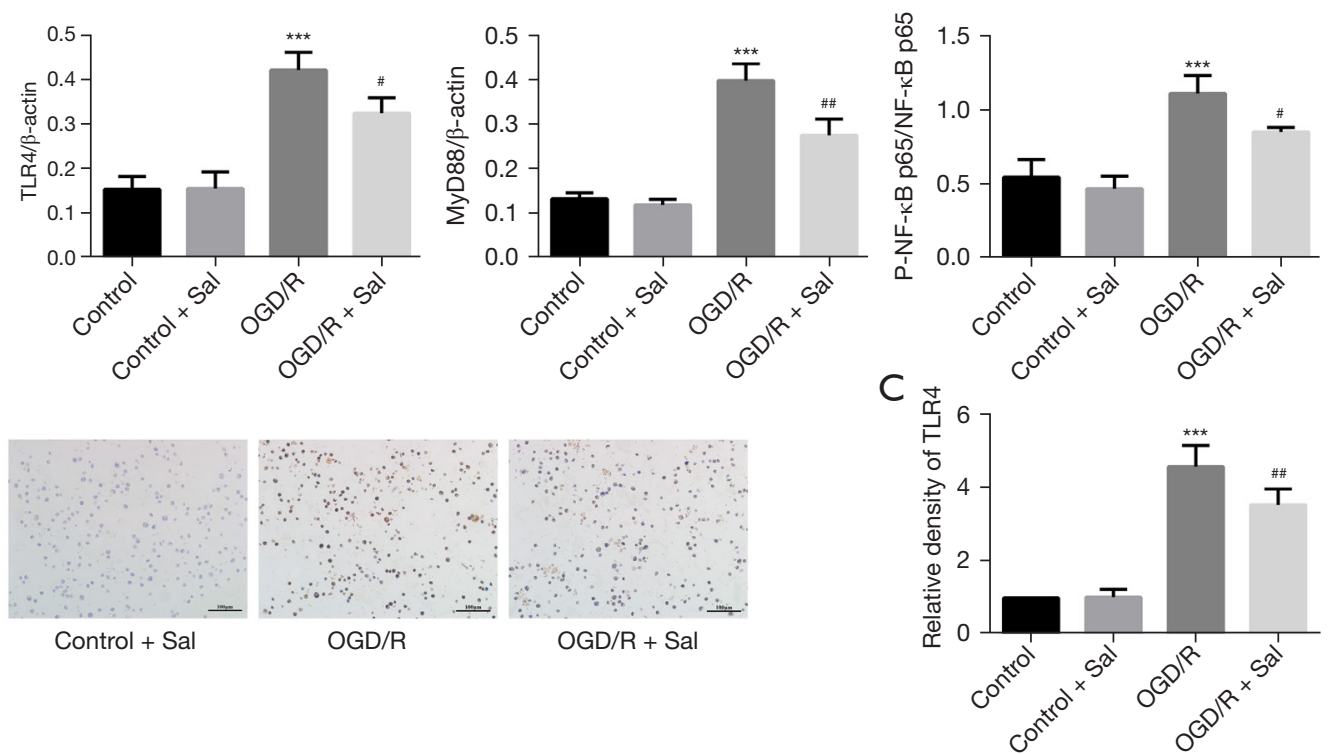

Figure $5 \mathrm{Sal}$ inhibited the TLR4/NF- $\mathrm{B}$ signaling pathway activation in OGD/R-induced BV2 cells. (A) Protein expression levels of TLR4,

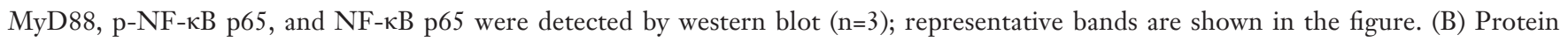
expression levels of TLR4 were detected by IHC staining (scale bar $=100 \mu \mathrm{m}$ ). (C) Quantitative analysis of IHC staining results ( $\mathrm{n}=5$ ). Data are expressed as mean $\pm \mathrm{SD}$. ${ }^{* * *} \mathrm{P}<0.001$ compared with control group; ${ }^{\#} \mathrm{P}<0.05,{ }^{\# \#} \mathrm{P}<0.01$ compared with OGD/R group. OGD, oxygen and glucose deprivation; TLR4, Toll-like receptor 4; MyD88, myeloid differentiation primary response 88; p-NF- $\mathrm{B}$ p65, phosphorylated nuclear factor kappa B p65; IHC, immunohistochemical; SD, standard deviation.

staining (Figure $7 F, 7 G$ ) and immunofluorescence staining (Figure $7 \mathrm{H}-77$ ) showed that the expression level of NLRP3 in microglia in the $\mathrm{MCAO} / \mathrm{R}+$ Sal group was decreased compared with that in the MCAO/R group. This showed that Sal can reduce the activation of NLRP3 inflammasomes in microglia induced by $\mathrm{MCAO} / \mathrm{R}$. This is consistent with the results of in vitro experiments. In conclusion, the in vivo experimental results further indicated that Sal may inhibit cell apoptosis and inhibit the activation of NLRP3 inflammasomes in microglia.

\section{Discussion}

In this study, we found that Sal could improve the viability of OGD/R-induced BV2 cells, reduce LDH release, and inhibit cell apoptosis. In addition, Sal inhibited NLRP3 inflammasome activation, decreased release of inflammatory factors, and inhibited the inflammatory response of OGD/ R-induced BV2 cells. These inhibitory effects of Sal may be achieved by inhibiting the TLR4/NF- $\kappa B$ signaling pathway in BV2 cells following induction by OGD/R. In the $\mathrm{MCAO} / \mathrm{R}$ rat model, we also found that Sal inhibited NLRP3 inflammasome activation in microglia, reduced the volume of cerebral infarction, and inhibited apoptosis. The results of this study provide a theoretical basis for the use of Sal as a neuroprotective drug in the treatment of cerebral ischemia.

The incidence of ischemic stroke is high, harm is great, and effective treatments are limited. The good curative effects of traditional Chinese medicine have attracted extensive attention from researchers. Sal is a biologically active extract mainly obtained from the traditional herbal medicine Rhodiola (34). Previous studies found that it has a good anti-inflammatory effect, but its specific antiinflammatory mechanism requires further study. Increasing evidence has indicated that inflammation is a major factor in the pathogenesis of cerebral ischemia (35). The main mechanism of inflammation in ischemic stroke is the activation of microglia, which further promote neuronal death (36). After cerebral ischemia, microglia become overactivated and release inflammatory cytokines such as IL- $1 \beta$, TNF- $\alpha$, IL- 6 , and IL-8, as well as cytotoxic molecules such as $\mathrm{NO}$ and reactive oxygen species, which cause nerve injury $(37,38)$. In addition, inflammatory or apoptotic mediators produced by overactivated microglia may cause and aggravate microglial apoptosis (39). The 
A

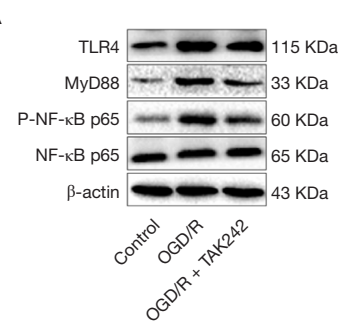

B

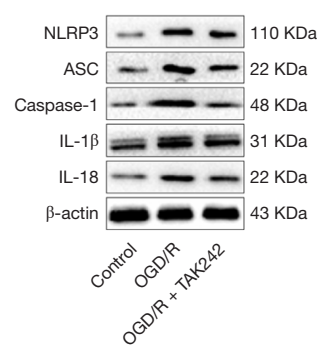

C

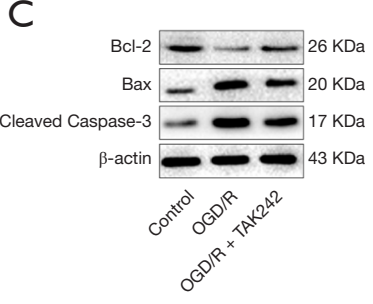

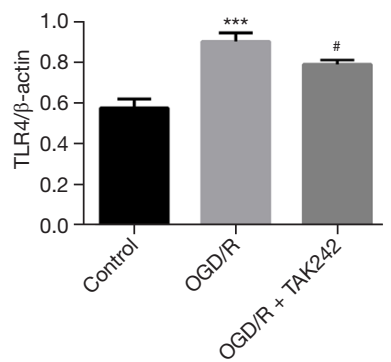
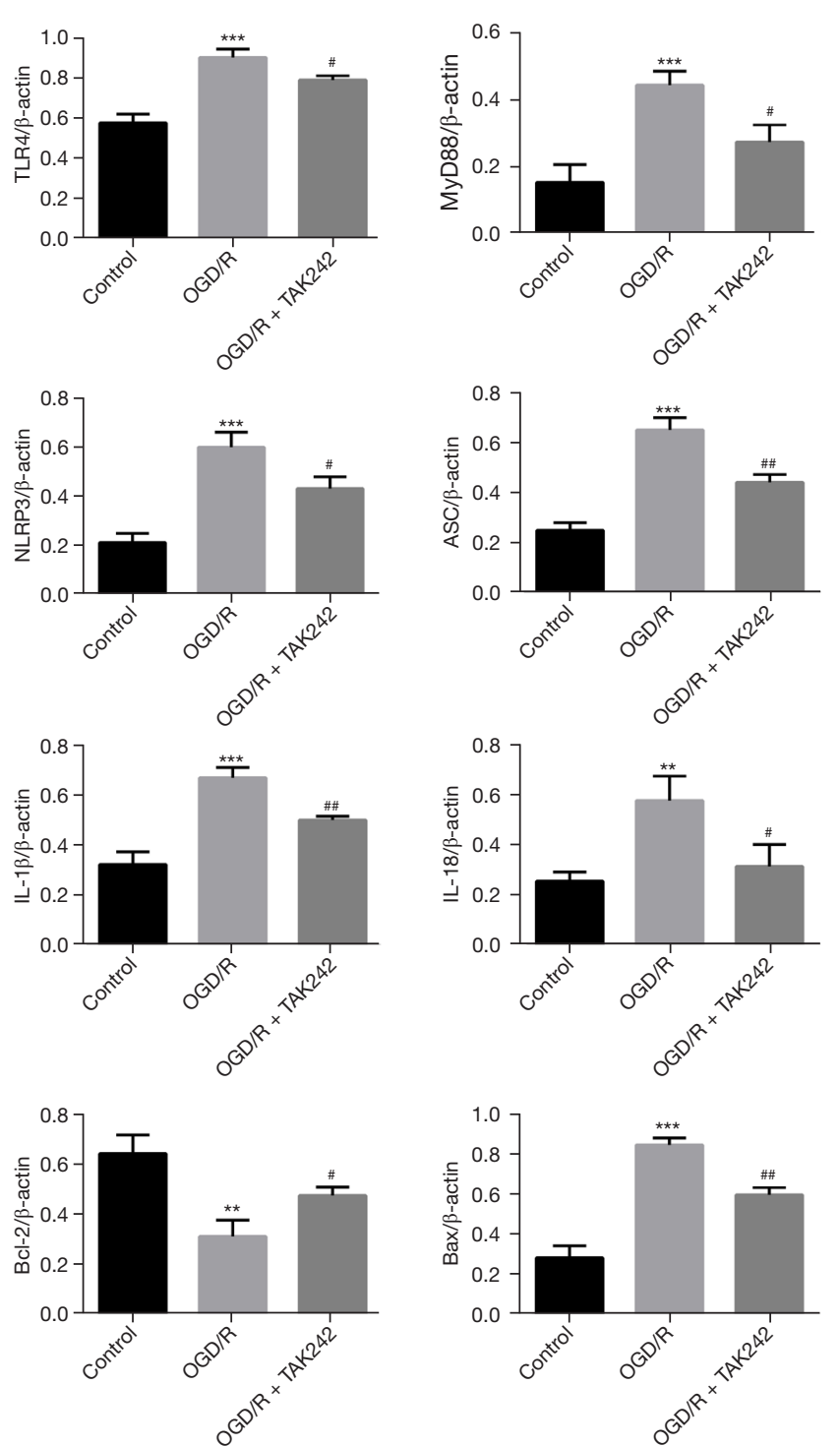
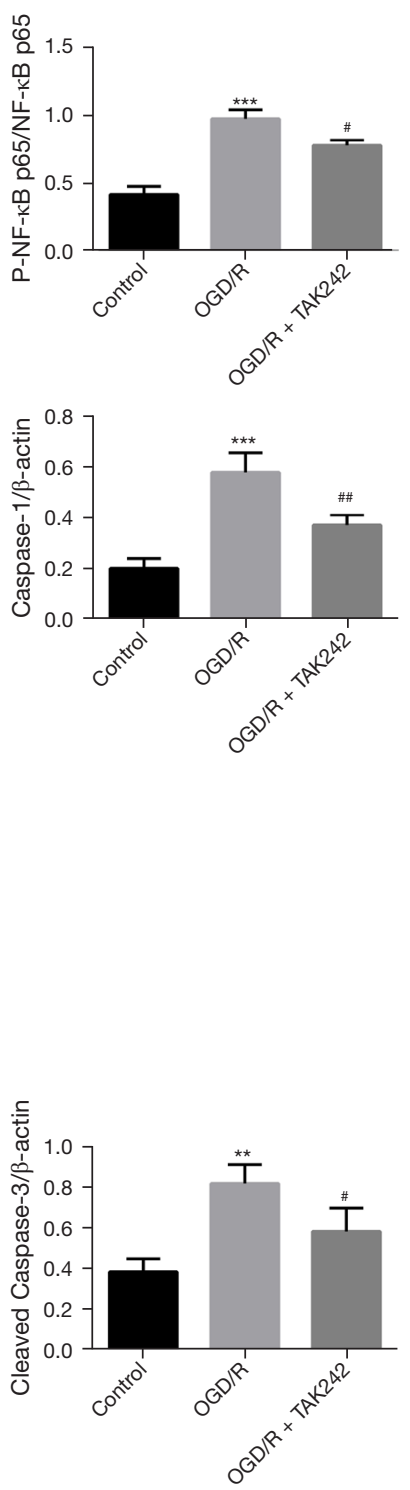

Figure 6 Inhibition of TLR4 reduced NLRP3 inflammasome activation and apoptosis in OGD/R-induced BV2 cells by inhibiting the TLR4/NF-אB signaling pathway. OGD/R-induced BV2 cells were treated with a TLR4 inhibitor, and western blot (n=3) was used to detect

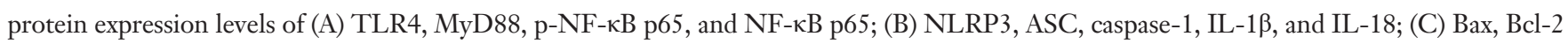
and cleaved caspase- 3 . Representative western blot bands are shown in the figure. Data are expressed as mean $\pm \mathrm{SD}$. ${ }^{* *} \mathrm{P}<0.01,{ }^{* * *} \mathrm{P}<0.001$ compared with control group; ${ }^{\prime} \mathrm{P}<0.05,{ }^{, \#} \mathrm{P}<0.01$ compared with OGD/R group. OGD, oxygen and glucose deprivation; TLR4, Toll-like receptor 4; MyD88, myeloid differentiation primary response 88; p-NF-kB p65, phosphorylated nuclear factor kappa B p65; ASC, apoptosisassociated speck-like protein containing a CARD; NLRP3, NOD-like receptor family pyrin domain-containing 3; SD, standard deviation.

main components of apoptotic pathways include the caspase and $\mathrm{Bcl}-2$ protein families (40). Caspase 3 is the key executor of cell apoptosis, while overexpression of Bax promotes cell apoptosis; formation of a heterodimer between Bax and Bcl-2 inhibits the pro-death effect of Bax (41). The relationship between microglial apoptosis and inflammatory response is still unclear. A previous study suggested that inhibiting OGD-induced apoptosis of microglia can inhibit OGD-induced microglial inflammatory responses (42). Another study suggested that promoting M2 polarization of microglia could reduce OGD/R-induced cell inflammation and apoptosis (13). In addition, eicosapentaenoic acid can reportedly inhibit both the activation of caspase- 3 and NLRP3, thereby reducing OGD-induced apoptosis 
A

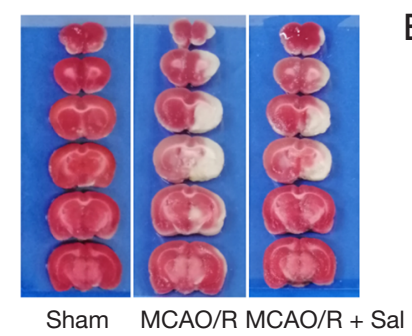

$\mathrm{D}$

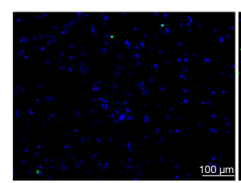

Sham

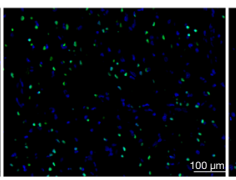

$\mathrm{MCAO} / \mathrm{R}$
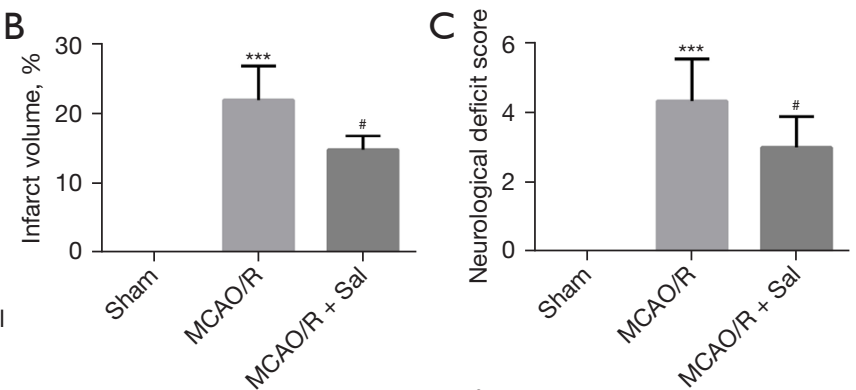

$\mathrm{E} \frac{0}{\mathrm{~d}}$

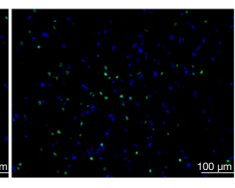

$\mathrm{MCAO} / \mathrm{R}+\mathrm{Sal}$

$\mathrm{F}$
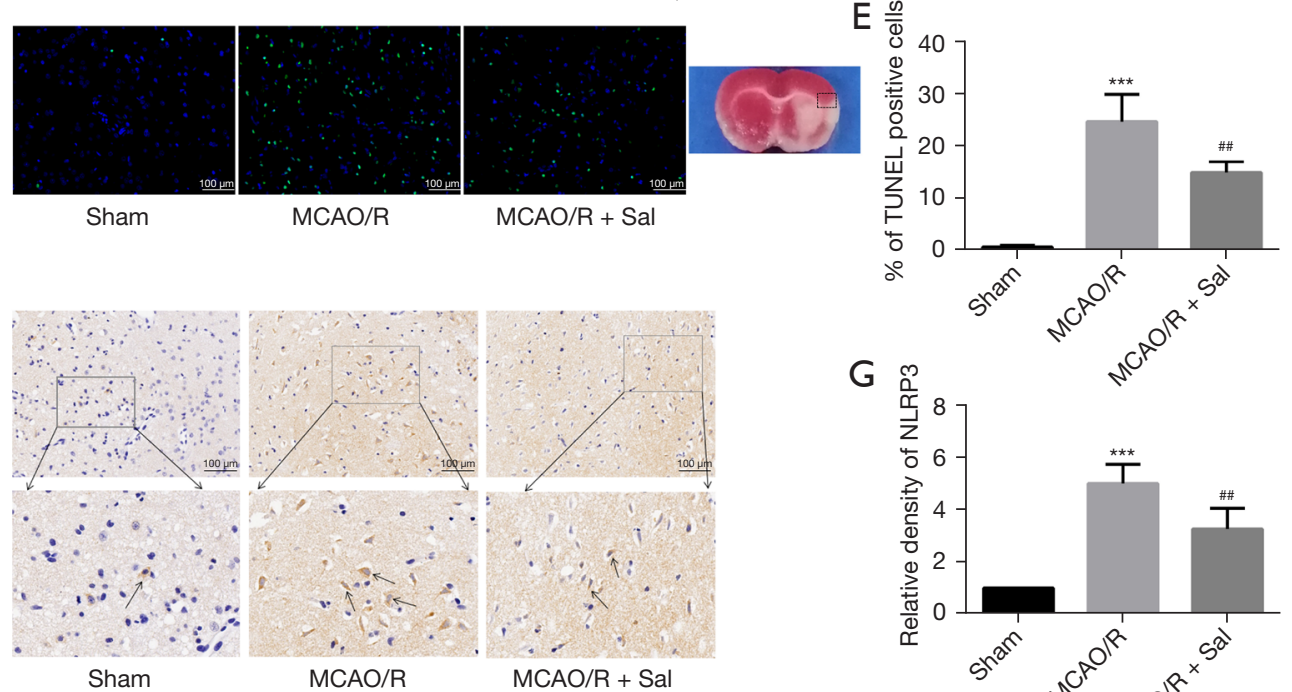

G

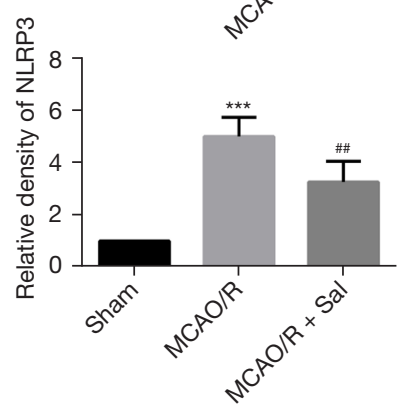

$\mathrm{H}$

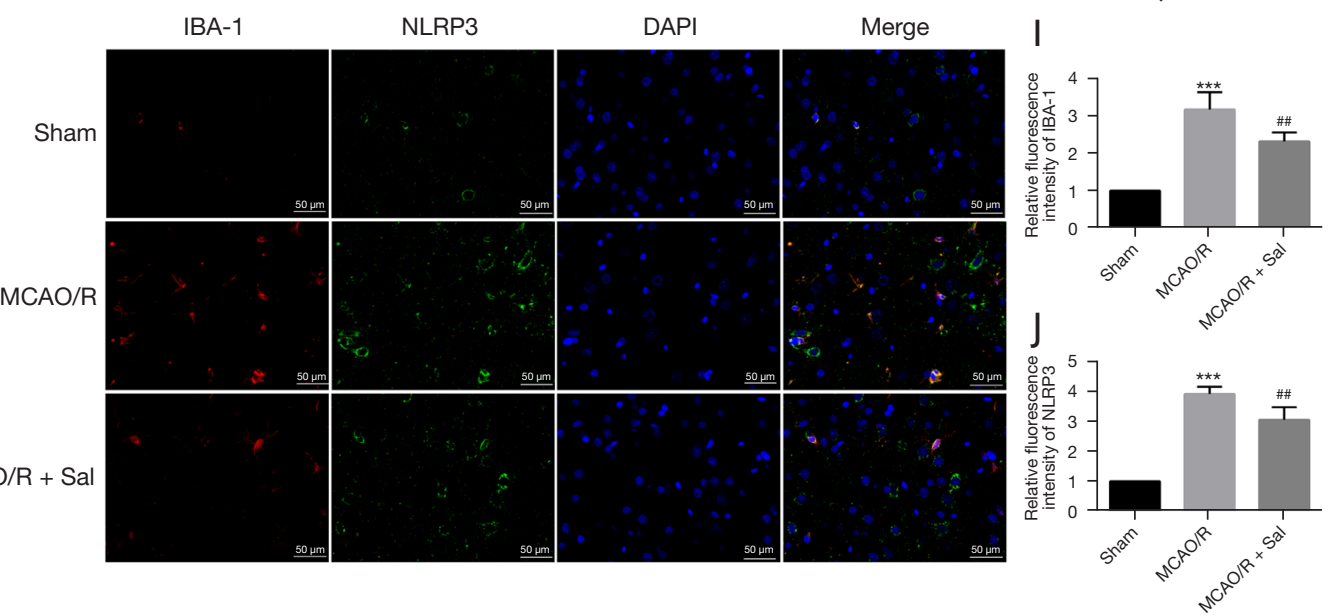

Figure $7 \mathrm{Sal}$ inhibited brain injury induced by MCAO/R in rats and inhibited the NLRP3 inflammasome activation in microglia. $(A, B)$ TTC staining was used to detect cerebral infarction volume in rats. A representative image is shown here, with infarcts in white $(n=4)$. (C) Neurological deficits were detected by the mNSS (n=9). (D,E) TUNEL staining was used to detect cell apoptosis in ischemic penumbra of brain tissue ( $\mathrm{n}=5$ ). DAPI (blue), TUNEL positive staining (green), scale bar $=100 \mu \mathrm{m}$. (F,G) The expression level of NLRP3 protein in brain tissues was detected by IHC staining $(\mathrm{n}=5)$ (scale bar $=100 \mu \mathrm{m})$. (H-J) The protein expression levels of NLRP3 and IBA- 1 in brain tissues were detected by immunofluorescence staining $(\mathrm{n}=5)$ (scale bar $=50 \mu \mathrm{m})$. Data are expressed as mean $\pm \mathrm{SD}$. ${ }^{* * *} \mathrm{P}<0.001$, compared with sham group; ${ }^{\prime \prime} \mathrm{P}<0.05,{ }^{\# \prime} \mathrm{P}<0.01$ compared with $\mathrm{MCAO} / \mathrm{R}$ group. $\mathrm{MCAO} / \mathrm{R}$ group, middle cerebral artery occlusion/reperfusion; NLRP3, NOD-like receptor family pyrin domain-containing 3; TTC, 2,3,5-triphenyltetrazolium chloride; mNSS, modified Neurological Severity Score; IHC, immunohistochemical; TUNEL, terminal deoxynucleotidyl transferase dUTP nick-end labeling assay; SD, standard deviation. 
of BV2 cells (43). In short, the state of microglia has an important influence on the inflammatory response to cerebral ischemia. Therefore, we speculate that inhibiting the inflammatory injury produced by microglia is an effective strategy to reduce cerebral I/R injury. Previous studies have shown that Sal can inhibit the inflammatory response of BV2 cells induced by corticotropin-releasing hormone or lipopolysaccharide $(44,45)$. However, it has not been reported whether Sal can inhibit the inflammatory response and apoptosis of microglia induced by OGD/R. In this study, we found that Sal could inhibit the release of the inflammatory cytokines TNF- $\alpha$, IL-6, and IL-8 in OGD/ $\mathrm{R}$-induced BV2 cells, thus inhibiting their inflammatory response. In addition, we found that Sal could reduce the percentage of apoptotic BV2 cells induced by OGD/ $\mathrm{R}$. Thus, we hypothesized that Sal plays a protective role by inhibiting the inflammatory response and apoptosis of OGD/R-induced BV2 cells.

Currently, the mechanism by which Sal exerts a protective effect on OGD/R-induced microglia is still unclear. Previous studies have shown that the NLRP3 inflammasome is a key mediator of cerebral ischemic inflammatory injury and obviously activated after cerebral I/R, whereby it promoted the release of inflammatory mediators and caused brain injury. However, inhibiting NLRP3 inflammasome activation can reduce brain injury by inhibiting the inflammatory response (46). The NLRP3 inflammasomes of microglia were activated after OGD/ $\mathrm{R}$ induction, but inhibition of NLRP3 inflammasome activation could curb the inflammatory response of OGD/ $\mathrm{R}$-induced microglia (47). Once the NLRP3 inflammasome is activated, it can form active caspase 1 , which mediates the release of inflammatory molecules IL-1 $\beta$ and IL-18 to cause inflammation (48). These studies have indicated that inhibition of NLRP3 inflammasome activation may be a promising approach for the treatment of ischemic stroke. It has been suggested that NLRP3 inflammasome activation is mediated by the TLR4/NF- $\mathrm{BB}$ and NLRP3/ASC/caspase-1 signaling pathways (49). First, the NLRP3/ASC/caspase-1 signaling pathway is involved in activation of the NLRP3 inflammasome, including formation of NLRP3 within the inflammasome, self-cleavage of pre-caspase 1 , and maturation of IL-1 $\beta$ and IL-18 (17). In addition, NLRP3 inflammasome activation is closely related to activation of the TLR4/NF- $\kappa \mathrm{B}$ signaling pathway (50). When TLR4 binds to $\mathrm{MyD} 88$ protein and activates NF- $\mathrm{\kappa B}$, a subsequent cascade of inflammatory responses is elicited (51). Activated $\mathrm{NF}-\kappa \mathrm{B}$ is transported from the cytoplasm into the nucleus, whereby it promotes the secretion of pre-IL-1 $\beta$, pre-IL-18, and NLRP3, leading to an inflammatory response (52). Meisoindigo reportedly inhibits NLRP3 inflammasome activation through the TLR4/NF- $\mathrm{KB}$ signaling pathway, regulates microglia/macrophage polarization, and has a protective effect against cerebral I/R injury (53). However, it has remained unclear whether Sal can inhibit the inflammatory response to cerebral ischemia by inhibiting activation of the NLRP3 inflammasome in microglia. To answer this question, we conducted related biomolecular studies, which demonstrated that Sal inhibited activation of the NLRP3 inflammasome and TLR4 signaling pathway in ODG/R-induced BV2 cells, thereby inhibiting the inflammatory response.

Mainly expressed by microglia, TLR4 plays a key role in innate immunity of the central nervous system (54). The TLR4/NF- $\kappa B$ signaling pathway is activated under conditions of ischemia and hypoxia, causing inflammatory injury; whereas, inhibition of the TLR4/NF- $\kappa B$ signaling pathway can inhibit inflammatory responses and apoptosis (55). Previous studies have found that TLR4 is significantly activated in cerebral I/R injury models, but TLR4 inhibitors can inhibit this phenomenon to reduce cerebral infarction volume and inflammatory cytokine expression (56). In addition, pretreatment with TLR4 inhibitors can reduce NF- $\mathrm{KB}$ signal transduction, thereby inhibiting microglia from producing pro-inflammatory cytokines such as IL-1 $\beta$, TNF- $\alpha$, and IL-6 (57). To further verify the regulatory relationship between the TLR4 signaling pathway, NLRP3 inflammasome activation, and apoptosis, OGD/R-induced BV2 cells were incubated with TAK242, a specific inhibitor of TLR4. Upon inhibiting TLR4 expression with TAK242, NLRP3 inflammasome activation and apoptosis were observed in OGD/R-induced BV2 cells. Thus, TAK242 could simultaneously inhibit the TLR4 signaling pathway, NLRP3 inflammasome activation, and apoptosis of OGD/R-induced BV2 cells. These results suggest that inhibition of TLR4 has the same effect as treatment with Sal. Thus, we speculate that the protective effect of Sal may be realized by reducing activation of the NLRP3 inflammasome and apoptosis through inhibition of the TLR4/NF- $\mathrm{kB}$ signaling pathway.

In this study, we preliminarily demonstrated the abovementioned phenomenon in an ischemic stroke rat model. We found that Sal reduced cerebral infarction volume and inhibited apoptosis of brain cells in the MCAO/ $\mathrm{R}$ rat model. Therefore, we hypothesized that Sal may also inhibit microglial apoptosis. In addition, IHC and 
immunofluorescence staining suggested that Sal could inhibit the NLRP3 inflammasome activation in microglia. However, the specific regulatory mechanism of this inhibitory effect of Sal in rats remains to be further studied. In conclusion, in vivo experiments further confirmed that Sal may inhibit NLRP3 inflammasome activation and apoptosis in microglia induced by cerebral I/R injury.

\section{Conclusions}

Through this study, we found that Sal inhibits NLRP3 inflammasome activation and apoptosis in microglia induced by cerebral I/R injury by a mechanism potentially related to inhibition of the TLR4/NF- $\mathrm{BB}$ signaling pathway. This study provides new theoretical support for Sal in the treatment of ischemic stroke.

\section{Acknowledgments}

Funding: This work was supported by the research innovation team of Yunnan province (2019HC022 to LYL) and the National Natural Science Foundation of China (31560295, 82160263 to LYL).

\section{Footnote}

Reporting Checklist: The authors have completed the ARRIVE reporting checklist. Available at https://dx.doi. org/10.21037/atm-21-5752

Data Sharing Statement: Available at https://dx.doi. org/10.21037/atm-21-5752

Conflicts of Interest: All authors have completed the ICMJE uniform disclosure form (available at https://dx.doi. org/10.21037/atm-21-5752). The authors have no conflicts of interest to declare.

Ethical Statement: The authors are accountable for all aspects of the work in ensuring that questions related to the accuracy or integrity of any part of the work are appropriately investigated and resolved. All rat experiments were approved by the Animal Research Ethics Committee of Kunming Medical University (Kmmu202100001410) and carried out in accordance with the Guide for the Care and Use of Laboratory Animals, 8th edition.

Open Access Statement: This is an Open Access article distributed in accordance with the Creative Commons Attribution-NonCommercial-NoDerivs 4.0 International License (CC BY-NC-ND 4.0), which permits the noncommercial replication and distribution of the article with the strict proviso that no changes or edits are made and the original work is properly cited (including links to both the formal publication through the relevant DOI and the license). See: https://creativecommons.org/licenses/by-nc-nd/4.0/.

\section{References}

1. Ajoolabady A, Wang S, Kroemer G, et al. Targeting autophagy in ischemic stroke: From molecular mechanisms to clinical therapeutics. Pharmacol Ther 2021;225:107848.

2. Xu Q, Guohui M, Li D, et al. IncRNA C2dat2 facilitates autophagy and apoptosis via the miR-30d-5p/DDIT4/ mTOR axis in cerebral ischemia-reperfusion injury. Aging (Albany NY) 2021;13:11315-35.

3. Zhang P, Cui J. Neuroprotective Effect of Fisetin Against the Cerebral Ischemia-Reperfusion Damage via Suppression of Oxidative Stress and Inflammatory Parameters. Inflammation 2021;44:1490-506.

4. Duris K, Jurajda M. Evolutionary concept of inflammatory response and stroke. J Neurosci Res 2020;98:98-104.

5. Wierońska JM, Cieślik P, Kalinowski L. Nitric OxideDependent Pathways as Critical Factors in the Consequences and Recovery after Brain Ischemic Hypoxia. Biomolecules 2021;11:1097.

6. Cao BQ, Tan F, Zhan J, et al. Mechanism underlying treatment of ischemic stroke using acupuncture: transmission and regulation. Neural Regen Res 2021;16:944-54.

7. Colonna M, Butovsky O. Microglia Function in the Central Nervous System During Health and Neurodegeneration. Annu Rev Immunol 2017;35:441-68.

8. He J, Liu J, Huang Y, et al. Oxidative Stress, Inflammation, and Autophagy: Potential Targets of Mesenchymal Stem Cells-Based Therapies in Ischemic Stroke. Front Neurosci 2021;15:641157.

9. Yu F, Huang T, Ran Y, et al. New Insights Into the Roles of Microglial Regulation in Brain Plasticity-Dependent Stroke Recovery. Front Cell Neurosci 2021;15:727899.

10. Wang K, Ru J, Zhang H, et al. Melatonin Enhances the Therapeutic Effect of Plasma Exosomes Against Cerebral Ischemia-Induced Pyroptosis Through the TLR4/NF- $\mathrm{kB}$ Pathway. Front Neurosci 2020;14:848.

11. Lu Y, Lou J, Liu X, et al. Oxysophocarpine reduces oxygen-glucose deprivation-induced microglial activation and injury. Am J Transl Res 2017;9:2266-75. 
12. Zhang X, Feng Y, Li J, et al. MicroRNA-665-3p attenuates oxygen-glucose deprivation-evoked microglial cell apoptosis and inflammatory response by inhibiting NF- $\mathrm{NB}$ signaling via targeting TRIM8. Int Immunopharmacol 2020;85:106650.

13. Li C, Fan C, Zhao J, et al. Panaxatriol Saponins Promote M2 Polarization of BV2 Cells to Reduce Inflammation and Apoptosis after Glucose/Oxygen Deprivation by Activating STAT3. Inflammation 2020;43:2109-18.

14. Lv Y, Cao H, Chu L, et al. Effects of Gastrodin on BV2 cells under oxygen-glucose deprivation and its mechanism. Gene 2021;766:145152.

15. Shichita T. Molecular and cellular mechanisms underlying the sterile inflammation after ischemic stroke. Nihon Yakurigaku Zasshi 2018;151:9-14.

16. Martinon F, Burns K, Tschopp J. The inflammasome: a molecular platform triggering activation of inflammatory caspases and processing of proIL-beta. Mol Cell 2002;10:417-26.

17. Latz E, Xiao TS, Stutz A. Activation and regulation of the inflammasomes. Nat Rev Immunol 2013;13:397-411.

18. Alishahi M, Farzaneh M, Ghaedrahmati F, et al. NLRP3 inflammasome in ischemic stroke: As possible therapeutic target. Int J Stroke 2019;14:574-91.

19. Franke M, Bieber M, Kraft P, et al. The NLRP3 inflammasome drives inflammation in ischemia/reperfusion injury after transient middle cerebral artery occlusion in mice. Brain Behav Immun 2021;92:223-33.

20. Peng J, Wang H, Gong Z, et al. Idebenone attenuates cerebral inflammatory injury in ischemia and reperfusion via dampening NLRP3 inflammasome activity. Mol Immunol 2020;123:74-87.

21. Sahin E, Bektur E, Burukoglu Donmez D, et al. Mirtazapine suppresses sterile inflammation through NLRP3-inflammasome in diabetic rat kidney. Acta Histochem 2019;121:289-96.

22. Zhong X, Liu M, Yao W, et al. Epigallocatechin-3-Gallate Attenuates Microglial Inflammation and Neurotoxicity by Suppressing the Activation of Canonical and Noncanonical Inflammasome via TLR4/NF-кB Pathway. Mol Nutr Food Res 2019;63:e1801230.

23. Huang D, Zhou J, Li W, et al. Casticin protected against neuronal injury and inhibited the TLR4/NF- $\kappa$ B pathway after middle cerebral artery occlusion in rats. Pharmacol Res Perspect 2021;9:e00752.

24. Wu M, Liu F, Guo Q. Quercetin attenuates hypoxiaischemia induced brain injury in neonatal rats by inhibiting TLR4/NF- $\kappa \mathrm{B}$ signaling pathway. Int Immunopharmacol 2019;74:105704.
25. Hu R, Wang MQ, Ni SH, et al. Salidroside ameliorates endothelial inflammation and oxidative stress by regulating the AMPK/NF-кB/NLRP3 signaling pathway in AGEsinduced HUVECs. Eur J Pharmacol 2020;867:172797.

26. Zhang $X$, Lai $W$, Ying $X$, et al. Salidroside Reduces Inflammation and Brain Injury After Permanent Middle Cerebral Artery Occlusion in Rats by Regulating PI3K/ PKB/Nrf2/NFкB Signaling Rather than Complement C3 Activity. Inflammation 2019;42:1830-42.

27. Zheng T, Wang Q, Bian F, et al. Salidroside alleviates diabetic neuropathic pain through regulation of the AMPK-NLRP3 inflammasome axis. Toxicol Appl Pharmacol 2021;416:115468.

28. Ystgaard MB, Sejersted Y, Løberg EM, et al. Early Upregulation of NLRP3 in the Brain of Neonatal Mice Exposed to Hypoxia-Ischemia: No Early Neuroprotective Effects of NLRP3 Deficiency. Neonatology 2015;108:211-9.

29. Fu C, Zhang X, Lu Y, et al. Geniposide inhibits NLRP3 inflammasome activation via autophagy in $\mathrm{BV}-2$ microglial cells exposed to oxygen-glucose deprivation/reoxygenation. Int Immunopharmacol 2020;84:106547.

30. Wang C, Lou Y, Xu J, et al. Endoplasmic Reticulum Stress and NF-кB Pathway in Salidroside Mediated Neuroprotection: Potential of Salidroside in Neurodegenerative Diseases. Am J Chin Med 2017;45:1459-75.

31. Zhou K, Wu J, Chen J, et al. Schaftoside ameliorates oxygen glucose deprivation-induced inflammation associated with the TLR4/Myd88/Drp1-related mitochondrial fission in BV2 microglia cells. J Pharmacol Sci 2019;139:15-22.

32. National Research Council Committee for the Update of the Guide for the Care and Use of Laboratory Animals. The National Academies Collection: Reports funded by National Institutes of Health.Guide for the Care and Use of Laboratory Animals. Washington (DC): National Academies Press (US); 2011.

33. Zhang X, Du Q, Yang Y, et al. Salidroside alleviates ischemic brain injury in mice with ischemic stroke through regulating BDNK mediated PI3K/Akt pathway. Biochem Pharmacol 2018;156:99-108.

34. Fan F, Yang L, Li R, et al. Salidroside as a potential neuroprotective agent for ischemic stroke: a review of sources, pharmacokinetics, mechanism and safety. Biomed Pharmacother 2020;129:110458.

35. Dong X, Gao J, Zhang CY, et al. Neutrophil MembraneDerived Nanovesicles Alleviate Inflammation To Protect Mouse Brain Injury from Ischemic Stroke. ACS Nano 2019;13:1272-83.

36. Hu H, Li Z, Zhu X, et al. Salidroside Reduces Cell 


\section{Page 16 of 16}

Mobility via NF- $\kappa B$ and MAPK Signaling in LPSInduced BV2 Microglial Cells. Evid Based Complement Alternat Med 2014;2014:383821.

37. Qiu Y, Yin Q, Fei Y, et al. JLX001 Modulated the Inflammatory Reaction and Oxidative Stress in $\mathrm{pMCAO}$ Rats via Inhibiting the TLR2/4-NF-кB Signaling Pathway. Neurochem Res 2019;44:1924-38.

38. Xiang J, Zhang X, Fu J, et al. USP18 Overexpression Protects against Focal Cerebral Ischemia Injury in Mice by Suppressing Microglial Activation. Neuroscience 2019;419:121-8.

39. Xiang HF, Cao DH, Yang YQ, et al. Isoflurane protects against injury caused by deprivation of oxygen and glucose in microglia through regulation of the Toll-like receptor 4 pathway. J Mol Neurosci 2014;54:664-70.

40. Chelyshev IuA, Cherepnev GV, Săttkulov KI. Apoptosis in the nervous system. Ontogenez 2001;32:118-29.

41. Merry DE, Korsmeyer SJ. Bcl-2 gene family in the nervous system. Annu Rev Neurosci 1997;20:245-67.

42. Yao Z, Liu N, Zhu X, et al. Subanesthetic isoflurane abates ROS-activated MAPK/NF- $\mathrm{kB}$ signaling to repress ischemia-induced microglia inflammation and brain injury. Aging (Albany NY) 2020;12:26121-39.

43. Mo Z, Tang C, Li H, et al. Eicosapentaenoic acid prevents inflammation induced by acute cerebral infarction through inhibition of NLRP3 inflammasome activation. Life Sci 2020;242:117133.

44. Borgonetti V, Governa P, Biagi M, et al. Rhodiola rosea L. modulates inflammatory processes in a $\mathrm{CRH}$-activated BV2 cell model. Phytomedicine 2020;68:153143.

45. Liu X, Wen S, Yan F, et al. Salidroside provides neuroprotection by modulating microglial polarization after cerebral ischemia. J Neuroinflammation 2018;15:39.

46. Feng YS, Tan ZX, Wang MM, et al. Inhibition of NLRP3 Inflammasome: A Prospective Target for the Treatment of Ischemic Stroke. Front Cell Neurosci 2020;14:155.

47. Zhao J, Piao X, Wu Y, et al. Cepharanthine attenuates cerebral ischemia/reperfusion injury by reducing NLRP3 inflammasome-induced inflammation and oxidative stress via inhibiting 12/15-LOX signaling. Biomed Pharmacother 2020;127:110151.

48. Spalinger MR, Lang S, Gottier C, et al. PTPN22 regulates NLRP3-mediated IL1B secretion in an autophagydependent manner. Autophagy 2017;13:1590-601.

49. Li X, Shi MQ, Chen C, et al. Phthalide derivative CD21 ameliorates ischemic brain injury in a mouse model of global cerebral ischemia: involvement of inhibition of NLRP3. Int Immunopharmacol 2020;86:106714.
50. El-Sisi AEE, Sokar SS, Shebl AM, et al. Octreotide and melatonin alleviate inflammasome-induced pyroptosis through inhibition of TLR4-NF-kB-NLRP3 pathway in hepatic ischemia/reperfusion injury. Toxicol Appl Pharmacol 2021;410:115340.

51. Liu FY, Cai J, Wang C, et al. Fluoxetine attenuates neuroinflammation in early brain injury after subarachnoid hemorrhage: a possible role for the regulation of TLR4/ MyD88/NF- $\mathrm{kB}$ signaling pathway. J Neuroinflammation 2018;15:347.

52. Zhang X, Zhang Y, Li R, et al. Salidroside ameliorates Parkinson's disease by inhibiting NLRP3-dependent pyroptosis. Aging (Albany NY) 2020;12:9405-26.

53. Ye $\mathrm{Y}$, Jin T, Zhang $\mathrm{X}$, et al. Meisoindigo Protects Against Focal Cerebral Ischemia-Reperfusion Injury by Inhibiting NLRP3 Inflammasome Activation and Regulating Microglia/Macrophage Polarization via TLR4/NF- $\kappa B$ Signaling Pathway. Front Cell Neurosci 2019;13:553.

54. Lehnardt S, Lachance C, Patrizi S, et al. The toll-like receptor TLR4 is necessary for lipopolysaccharideinduced oligodendrocyte injury in the CNS. J Neurosci 2002;22:2478-86.

55. Zhang J, Xiao F, Zhang L, et al. Alpha-Lipoic Acid Preconditioning and Ischaemic Postconditioning Synergistically Protect Rats from Cerebral Injury Induced by Ischemia and Reperfusion Partly via Inhibition TLR4/ MyD88/ NF-кB Signaling Pathway. Cell Physiol Biochem 2018;51:1448-60.

56. Hua F, Tang H, Wang J, et al. TAK-242, an antagonist for Toll-like receptor 4, protects against acute cerebral ischemia/reperfusion injury in mice. J Cereb Blood Flow Metab 2015;35:536-42.

57. Fu S, Wang J, Hao C, et al. Tetramethylpyrazine ameliorates depression by inhibiting TLR4NLRP3 inflammasome signal pathway in mice. Psychopharmacology (Berl) 2019;236:2173-85.

(English Language Editor: J. Jones)

Cite this article as: Liu J, Ma W, Zang CH, Wang GD, Zhang SJ, Wu HJ, Zhu KW, Xiang XL, Li CY, Liu KP, Guo JH, Li LY. Salidroside inhibits NLRP3 inflammasome activation and apoptosis in microglia induced by cerebral ischemia/reperfusion injury by inhibiting the TLR4/NFкB signaling pathway. Ann Transl Med 2021;9(22):1694. doi: 10.21037/atm-21-5752 